\title{
Characterization of Major and Clinically Relevant Non-Major Bleeds in the APEX Trial
}

\author{
Megan K. Yee ${ }^{1}$ C. Michael Gibson ${ }^{1}$ Tarek Nafee ${ }^{1} \quad$ Mathieu Kerneis $^{2}$ Yazan Daaboul ${ }^{2}$ Serge Korjian ${ }^{2}$ \\ Gerald Chi ${ }^{2}$ Fahad AlKhalfan ${ }^{2}$ Adrian F. Hernandez ${ }^{3}$ Russell D. Hull ${ }^{4}$ Alexander T. Cohen ${ }^{5}$ \\ Samuel Z. Goldhaber ${ }^{6}$
}

\footnotetext{
${ }^{1}$ Boston Clinical Research Institute, Newton, Massachusetts, United States

2 PERFUSE Study Group, Division of Cardiovascular, Departments of Medicine, Beth Israel Deaconess Medical Center, Harvard Medical School, Boston, Massachusetts, United States

${ }^{3}$ Duke University and Duke Clinical Research Institute, Durham, North Carolina, United States

${ }^{4}$ Division of Cardiology, R.A.H. Faculty of Medicine, University of Calgary, Calgary, Alberta, Canada

${ }^{5}$ Department of Haematological Medicine, Guy's and St Thomas'

Hospitals, London, United Kingdom

6 Division of Cardiovascular, Brigham and Women's Hospital, Harvard

Medical School, Boston, Massachusetts, United States
}

Address for correspondence Megan K. Yee, MPH, Boston Clinical Research Institute, 1340 Centre Street, Suite 203, Newton, MA 02459, United States (e-mail: megan.yee19@gmail.com).

TH Open 2019;3:e103-e108.

\section{Abstract \\ Keywords \\ - venous thromboembolism \\ - betrixaban \\ - acutely ill patients \\ - major bleed \\ - clinically relevant non-major bleeding \\ Background Among medically ill patients treated with thromboprophylaxis, betrix- aban was not associated with an increase in major bleeding compared with enoxaparin, but an increase in clinically relevant non-major (CRNM) bleeding was observed. The aim of this analysis is to describe the severity and clinical consequences of major and CRNM bleeding in the APEX trial. \\ Methods The APEX trial randomized 7,513 hospitalized acutely ill medical patients to receive either enoxaparin for 6 to 14 days or betrixaban for 35 to 42 days. Subjects receiving a concomitant strong $\mathrm{p}$-glycoprotein inhibitor or with creatinine clearance $<30 \mathrm{~mL} / \mathrm{min}$ were administered a reduced dose of study drug. \\ Results A total of $25(0.7 \%)$ and $21(0.6 \%)$ major bleeds occurred in the betrixaban and enoxaparin arms, respectively $(p=N S)$ and a total of $91(2.5 \%)$ and $38(1.0 \%)$ CRNM bleeds occurred in the betrixaban and enoxaparin arm $(p<0.001)$, respectively. Most major bleeds were considered moderate or severe and most CRNM bleeds were considered mild and moderate $(p=N S)$. One fatal major bleed occurred in each treatment arm. Rates of major or CRNM bleeds resulting in new or prolonged hospitalization (major: 44.0 vs. $28.6 \%$; CRNM: 12.1 vs. $21.1 \%$ ) or study treatment interruption or cessation (major: 72.0 vs. $71.4 \%$; CRNM: 71.3 vs. $68.4 \%$ ) were similar between treatment arms ( $p=$ NS). \\ Conclusions In the APEX trial, CRNM bleeds were mild or moderate in nature and had less of a clinical impact than major bleeds. The severity and clinical sequela of bleeds in the betrixaban arm were comparable to those in the enoxaparin arm. \\ Clinical Trial Registration URL: http://www.clinicaltrials.gov.; Unique identifier: NCT01583218.}

(1)Dr. Gerald Chi's ORCID is https://orcid.org/0000-0002-8371-

1689.

received

December 20, 2018

accepted after revision

February 25, 2019
DOI https://doi.org/

10.1055/s-0039-1685496.

ISSN 2512-9465. (c) 2019 Georg Thieme Verlag KG Stuttgart · New York
License terms

(1)(1) 


\section{Introduction}

Acutely ill hospitalized patients are at an increased risk for venous thromboembolism (VTE) for at least a month after hospital discharge. ${ }^{1,2}$ However, current guidelines recommend the use of parenteral anticoagulants for 6 to 14 days, but do not recommend the use of extended-duration thromboprophylaxis. ${ }^{3}$ Trials of extended-duration thromboprophylaxis have demonstrated either a lack of efficacy or efficacy that has been accompanied by an increase in major bleeding. ${ }^{4-7}$ The APEX trial (Acute Medically Ill VTE Prevention with Extended Duration Betrixaban) demonstrated a significant reduction in a composite of asymptomatic deep vein thrombosis (DVT), symptomatic DVT, nonfatal pulmonary embolism (PE), and VTE-related death with extended duration betrixaban compared with standard duration enoxaparin. In contrast to prior studies, betrixaban was not associated with an increase in major bleeds, but was associated with an increase in clinically relevant non-major (CRNM) bleeds. ${ }^{8}$ The aim of this post hoc analysis is to describe the severity and clinical impact of major and CRNM bleeds observed in the APEX trial.

\section{Methods}

\section{Study Population and Design}

The APEX trial was a randomized, multicenter, double-blind, double-dummy, placebo-controlled, phase 3 clinical trial. The design and primary results have been previously published. ${ }^{8,9}$ Briefly, acutely ill medical patients aged 40 years or older with reduced mobility hospitalized within the last 96 hours for heart failure, respiratory failure, infectious disease, rheumatic disease, or ischemic stroke were eligible for inclusion. Major exclusion criteria included active bleeding, severe renal insufficiency defined as creatinine clearance $(\mathrm{CrCl})$ less than $15 \mathrm{~mL} / \mathrm{min}$, or any condition requiring long-term anticoagulation or antiplatelet therapy. Enrolled subjects were randomized in a 1:1 ratio to receive either (1) standard duration active $40 \mathrm{mg}$ enoxaparin once daily for 6 to 14 days plus extended duration oral placebo betrixaban for 35 to 42 days or (2) standard duration enoxaparin placebo for 6 to 14 days plus oral active $80 \mathrm{mg}$ betrixaban for 25 to 42 days. Subjects receiving a concomitant strong P-glycoprotein (P-gp) inhibitor or with renal insufficiency $(\mathrm{CrCl}$ $<30 \mathrm{~mL} / \mathrm{min}$ ) received a reduced dose of study drug $(40 \mathrm{mg})$.

\section{Bleeding Definitions in the APEX Trial}

In the main APEX trial, the primary safety endpoint was major bleeding and the secondary safety endpoint was the composite or major or clinically relevant non-major bleeding that occurred within 7 days of all study drug discontinuation. The current analysis will examine major bleeding and CRNM bleeding independently. Major bleeding was defined according to the International Society of Thrombosis and Haemostasis (ISTH) criteria, which includes a clinically overt bleeding that is fatal or associated with a reduction in hemoglobin of at least $2 \mathrm{~g} / \mathrm{dL}$, a transfusion of at least 2 units of blood or packed cells, or occurs in a critical area or organ (intraocular, intracranial, intraspinal, intramuscular with compartment syndrome, retroperitoneal bleeding, intraocular bleeding, or pericardial bleeding). ${ }^{10}$ CRNM bleeding was defined as an overt bleeding that did not meet the criteria for major bleeding, but was associated with a medical intervention, unscheduled contact with a physician (in person or by telephone), interruption or cessation of study treatment, or discomfort for the subject including pain or impairment of activities of daily living.

\section{Bleeding Severity}

Severity of events was investigator determined. Mild events were defined as events when the subject was aware of signs and symptoms, but the signs and symptoms were easily tolerated. Moderate events were defined as events that caused enough discomfort to interfere with activities of daily living. Severe events were defined as events that caused subjects to be unable to perform normal daily activities. Life-threatening events were those that posed an immediate risk of death.

\section{Bleeding-Related Clinical Outcomes}

Each bleed was assessed to determine if it resulted in any of the following four clinical outcomes: (1) death, (2) requiring or prolonging hospitalization, (3) requiring medical treatment, (4) causing study drug interruption, or (5) study drug cessation.

\section{Statistical Analysis}

Analyses were completed according to the study drug received and in the safety population which includes all subjects who received at least one dose of study drug. Major or CRNM bleeding was tallied in each treatment arm. If a subject experienced more than one bleed, the most severe bleed was counted. Descriptive analyses regarding the location, severity, and clinical outcomes of major and CRNM bleeds were completed according to study drug and dose. The chi-squared test of independence or Fisher's exact test, if any expected cell count was less than 5 , was used to test differences between treatment arms.

\section{Results}

\section{Study Population}

A total of 7,513 subjects were randomized in the APEX trial, with 3,759 subjects in the betrixaban arm and 3,754 subjects in the enoxaparin arm. Baseline characteristics were well balanced as previously reported. ${ }^{8}$ The safety population was composed of 7,432 subjects who received at least one dose of study drug, with 3,716 subjects in the betrixaban arm and 3,716 in the enoxaparin arm.

\section{Rates of Major and CRNM Bleeds}

As previously reported, the occurrence of major bleeding was similar between the betrixaban arm and the enoxaparin arm (betrixaban $=25[0.7 \%]$, enoxaparin $=21[0.6 \%], p=0.55$ ). Rates of major bleeding were unchanged among subjects who received the reduced dose (betrixaban $=10$ [1.4\%], enoxaparin $=5[0.7 \%], p=0.20$ ) and among subjects who received the full dose (betrixaban $=15[0.5 \%]$, enoxaparin $=16[0.5 \%]$, $p=0.86$ ). No subjects experienced more than one major 
bleed. An increase in CRNM bleeding was observed in the betrixaban arm with a total of 91 (2.5\%) bleeds compared with 38 (1.0\%) bleeds in the enoxaparin $\operatorname{arm}(p<0.001)$. Betrixaban was also associated with an increase in CRNM bleeding compared with enoxaparin among subjects who receive the reduced dose (betrixaban $=25[3.4 \%]$, enoxaparin $=5[0.7 \%]$, $p<0.001$ ) and the full dose (betrixaban $=66$ [2.2\%], enoxaparin $=33[1.1 \%], p<0.001)$. A total of three subjects in the betrixaban arm and one subject in the enoxaparin arm experienced more than one CRNM bleed. No subjects in either arm had both a major bleed and a CRNM bleed.

\section{Location of Bleeds}

The most common location of major bleeds was upper gastrointestinal (GI), which accounted for $72.0 \%(n=18 /$ 25 ) of the major bleeds in the betrixaban arm and 33.3\% $(n=7 / 21)$ in the enoxaparin $\operatorname{arm}(p=0.017)$. The second most common location for major bleeding was intracranial bleeding which accounted for $8.0 \%(n=2 / 25)$ in the betrixaban and $33.3 \%(n=7 / 21)$ in the enoxaparin arm $(p=0.059)$. Other locations which generally occurred in one patient per treatment arm included lower GI bleeding, hematomas, pericardial bleeding, rectal bleeding, bleeding associated with noncardiac surgery, epistaxis, and intraocular bleeding (- Fig. 1A; - Supplementary Table S1).

Locations of CRNM bleeds were comparable between treatment arms $(p>0.05)$. The most common bleed was gross hematuria accounting for $28.6 \%$ ( $n=26 / 91$ ) of CRNM bleeds in the betrixaban arm and 23.7\% $(n=9 / 38)$ in the enoxaparin arm. Epistaxis accounted for $16.5 \%(n=15 / 91)$ and $18.4 \%(n=7 / 38)$ CRNM bleeds in the betrixaban and enoxaparin arm, respectively. Other common locations included rectal bleeding (betrixaban $=11.0 \%[n=10 / 91]$, enoxaparin $=13.2 \%$ [ $n=5 / 38]$ ), upper GI bleeding (betrix- aban $=12.1 \%[n=11 / 91]$, enoxaparin $=10.5 \%[n=4 / 38])$, and hematomas (betrixaban $=8.8 \%[n=8 / 91]$, enoxaparin $=7.9 \%[n=3 / 38])$. All CRNM bleed locations are displayed in - Fig. 1B and - Supplementary Table S2.

\section{Severity of Bleeds}

Severity of major bleeding was similar between treatment arms $(p>0.05)$. Overall, $4.0 \%(n=1 / 25)$ of major bleeds in the betrixaban arm and 9.5\% $(n=2 / 21)$ of major bleeds in the enoxaparin arm were considered mild. Most major bleeds were considered moderate with $40.0 \%(n=10 / 25)$ and $33.3 \%$ ( $n=7 / 21)$ in the betrixaban and enoxaparin arm, respectively, or severe with $44.0 \%(n=11 / 25)$ in the betrixaban arm and $42.9 \%(n=9 / 21)$ in the enoxaparin arm. Major bleeds considered life threatening accounted for $12.0 \%$ $(n=3 / 25)$ in the betrixaban arm and $14.3 \%(n=3 / 21)$ in the enoxaparin arm. Results were unchanged among subjects receiving the reduced dose and the full dose with the majority of major bleeds considered moderate or severe (-Fig. 2A; - Supplementary Table S3).

Severity of CRNM bleeds was also similar between the betrixaban and enoxaparin treatment arms ( $p>0.05)$. Overall, the majority of CRNM bleeds were considered mild (betrixaban $=41.8 \%[n=38 / 91]$, enoxaparin $=44.7 \%[n=17 / 38])$ or moderate $\quad$ (betrixaban $=46.2 \% \quad[n=42 / 91]$, enoxaparin $=39.5 \%$, $[n=15 / 38])$. A low proportion of CRNM bleeds was considered severe with $12.1 \%(n=11 / 91)$ and $15.8 \%(n=6 / 38)$ in the betrixaban and enoxaparin arm, respectively, and no CRNM bleeds were considered life threatening. Results were consistent among subjects receiving the reduced dose and the full dose, with the majority of CRNM bleeds considered mild or moderate, a low proportion considered severe, and no bleeds considered life threatening ( - Fig. 2B; - Supplementary Table S4).
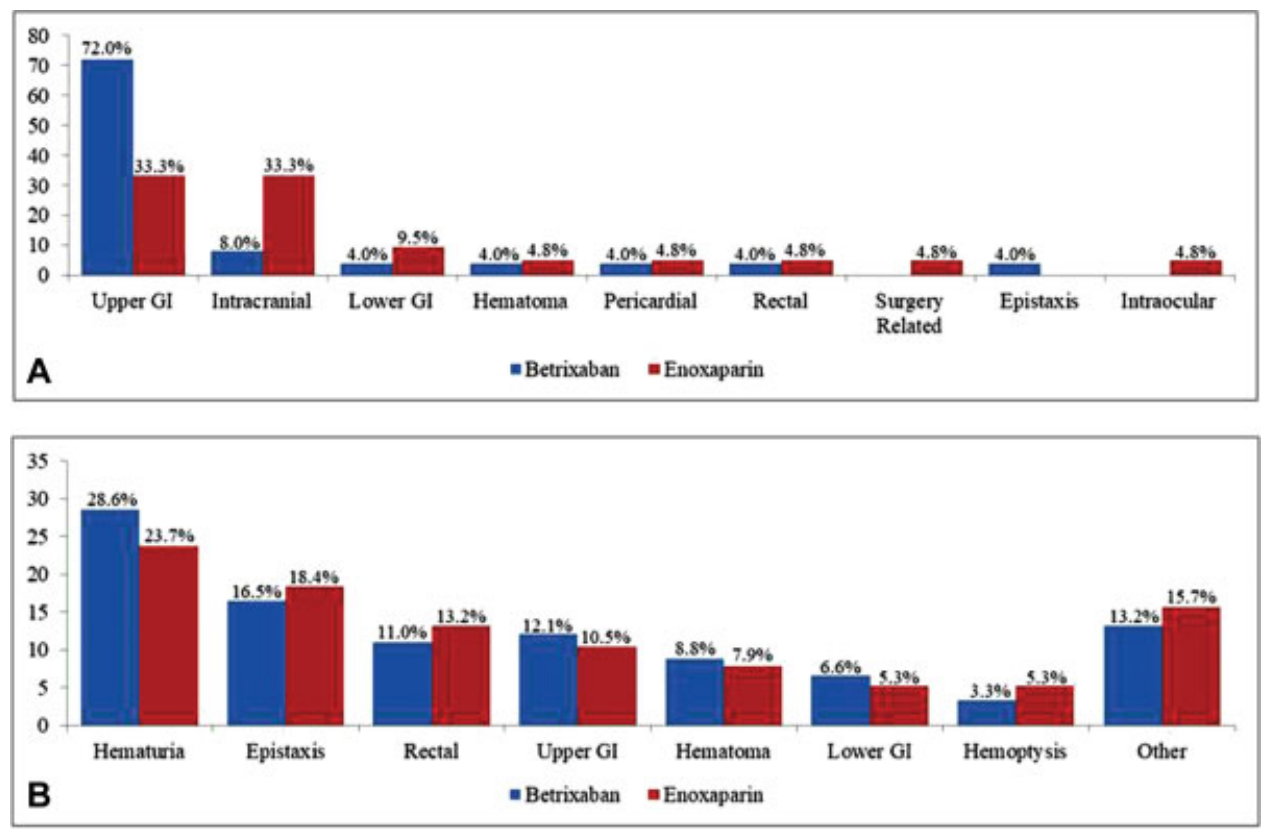

Fig. 1 Location of major bleeds (A) and location of CRNM bleeds (B). Other bleeds include laceration, hemothorax, vaginal (increased or prolonged menstrual or abnormal vaginal bleeding), bleeding associated with noncardiac surgery, gingival, puncture site, retroperitoneal, subconjunctival/ conjunctival, petechial rash/hemorrhage, baker's cyst with intra cyst hemorrhage, and prevertebral space posterior hypopharynx hematoma. 

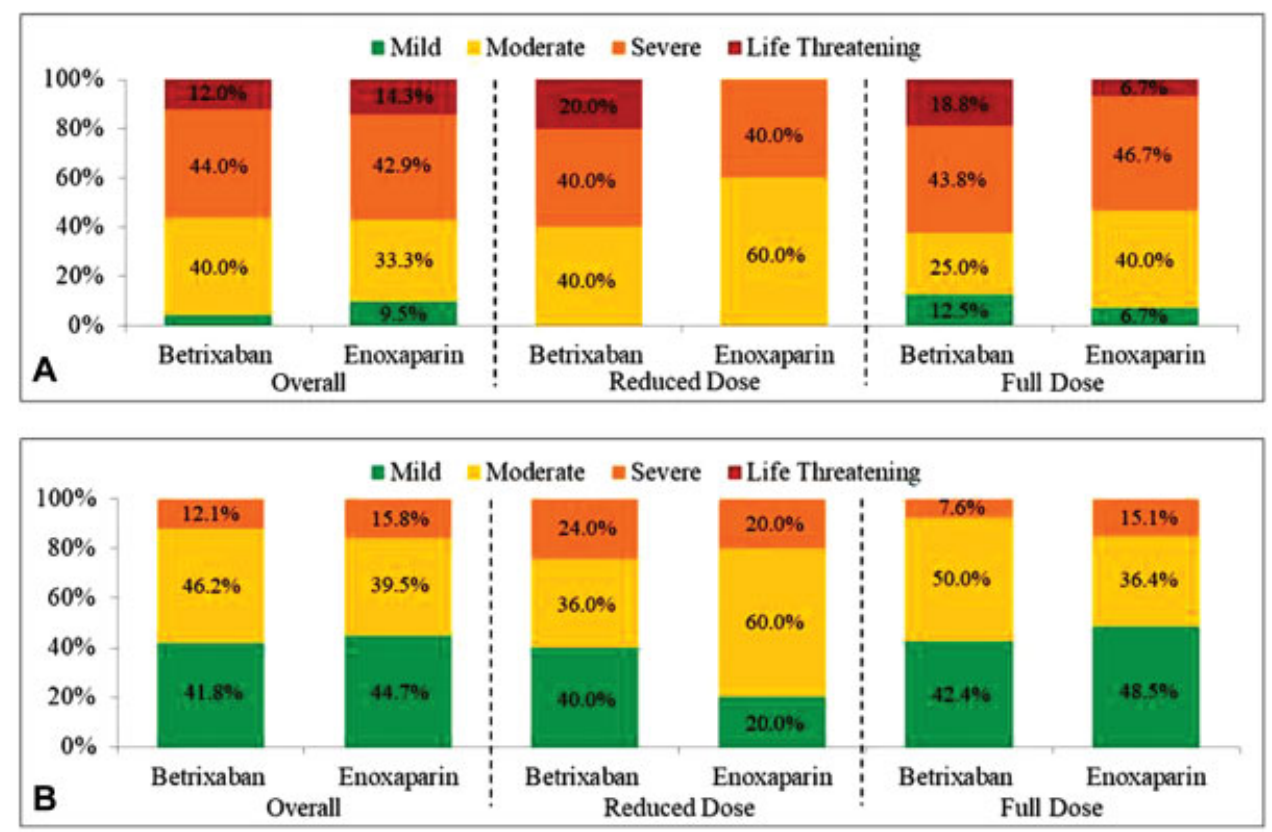

Fig. 2 Severity of major bleeds (A) and severity of CRNM bleeds (B).

\section{Clinical Outcomes of Bleeds}

One fatal bleed occurred in the betrixaban arm (4.0\%) and in the enoxaparin arm (4.8\%; $p>0.05)$. Approximately $44.0 \%$ ( $n=11 / 25)$ and $28.6 \%(n=6 / 21)$ of major bleeds resulted in a new or prolonged hospitalization $(p>0.05)$. Eighty-eight percent $(n=22 / 25)$ of major bleeds in the betrixaban arm and $61.9 \%(n=13 / 21)$ in the enoxaparin arm required medical treatment $(p=0.019)$. The majority of major bleeds resulted in study drug cessation (betrixaban $=60.0 \%$ $[n=15 / 25]$, enoxaparin $=71.4 \%[n=15 / 21])$ and a few major bleeds resulted in study drug interruption (betrixaban
$=12.0 \%[n=3 / 25])$. Results were unchanged among subjects receiving the reduced dose and the full dose (-Fig. 3A; -Supplementary Table S5).

No CRNM bleeds resulted in death in either treatment arm. Approximately $12.1 \%(n=11 / 91)$ of CRNM bleeds in the betrixaban arm and $21.1 \%(n=8 / 38)$ of CRNM bleeds in the enoxaparin arm resulted in a new or prolonged hospitalization $(p>0.05)$. CRNM bleeds required medical treatment in $47.3 \%$ $(n=43 / 91)$ of the betrixaban arm and $47.4 \%(n=18 / 38)$ of the enoxaparin arm $(p<0.05)$. Approximately half of CRNM bleeds resulted in study drug cessation in both treatment arms
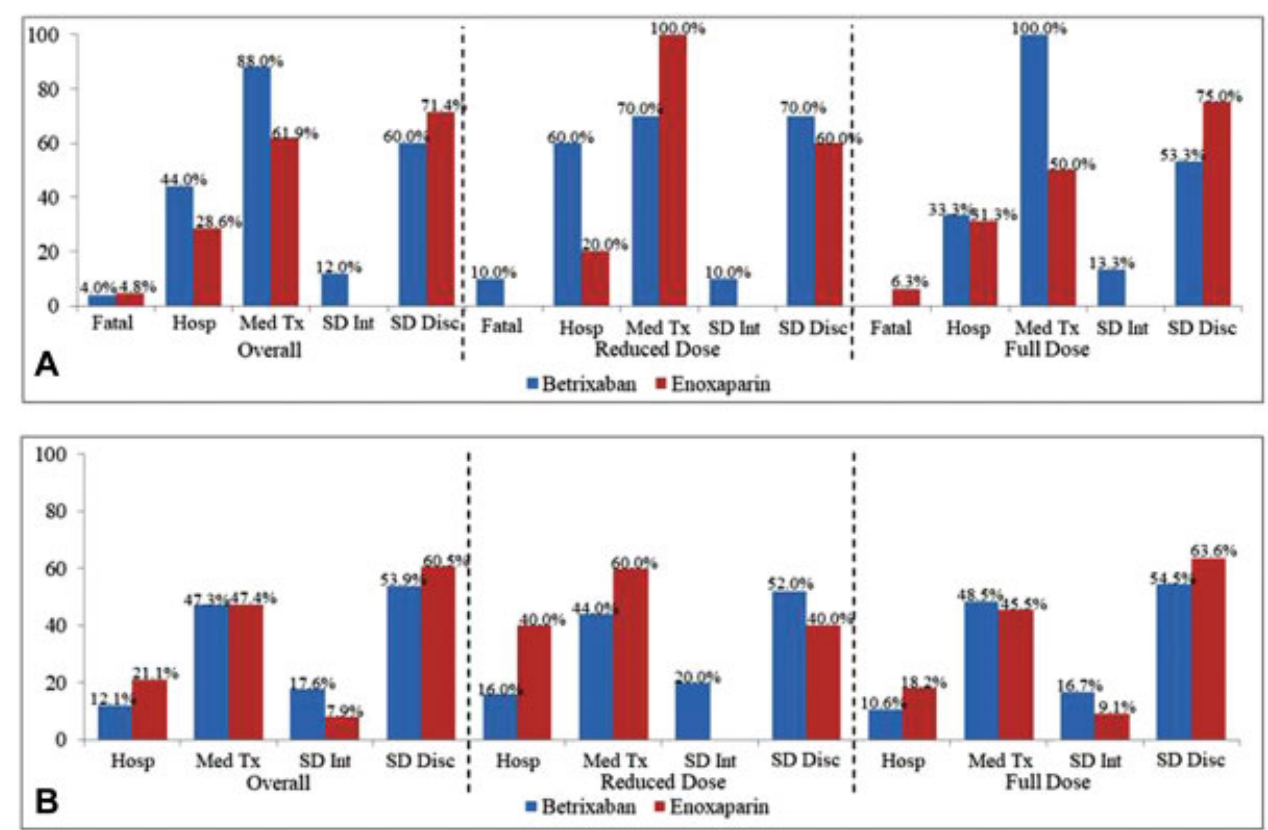

Fig. 3 Clinical outcomes of major bleeds (A) and clinical outcomes of CRNM bleeds (B). Hosp, required or prolonged hospitalization; Med Tx, required medical treatment; SD Int, study drug interruption; SD Disc, study drug cessation. 
(betrixaban $=53.9 \% \quad[n=49 / 91], \quad$ enoxaparin $=60.5 \%$ [ $n=23 / 38]$ ). Rate of study drug interruption was comparable between treatment arms (betrixaban $=17.6 \%$ [ $n=16 / 91]$, enoxaparin $=7.9 \%[n=3 / 38]$ ). Results remained the same among subjects receiving the reduced dose and the full dose (-Fig. 3B; -Supplementary Table S6).

\section{Discussion}

Bleeds experienced in the betrixaban arm were not more severe and did not result in worse clinical outcomes compared with enoxaparin, despite a longer duration of administration of betrixaban. Betrixaban was associated with more GI bleeds and enoxaparin tended to be associated with more intracranial bleeds. This trend of an increase in GI bleeds coupled with a decrease in intracranial bleeds has been previously observed with factor Xa inhibitors. ${ }^{11-14}$

Mitigating intracranial bleeding risk within the context of anticoagulation is of utmost importance given the incidence of anticoagulant-associated intracranial hemorrhages quadrupled over a single decade. ${ }^{15}$ Intracranial bleeds are a particular concern not only because of the associated high mortality rate, but also due to the associated burden on healthcare. Intracranial hemorrhage mortality rates are estimated to range between 50 and $57 \%,{ }^{16,17}$ though only two fatal bleeds occurred throughout the APEX trial duration, one in each treatment arm. Mean hospitalization costs per patient associated with mortality due to an intracranial bleed are estimated to be approximately $\$ 76,000$. Mean hospitalization costs associated with intracranial bleed survival are estimated even higher at approximately $\$ 119,000{ }^{18}$ In total, the lifetime cost within the U.S. healthcare system is approximately $\$ 6.0$ billion dollars. ${ }^{19}$

Severity patterns and clinical impacts of major and CRNM bleeds were unsurprising. Based on bleeding definitions alone, it is not unexpected that CRNM bleeds were milder and had less of a clinical impact compared with major bleeding. The APEX trial employed an especially sensitive definition for CRNM bleeding which included bleeds that could be reported via telephone without a face-to-face evaluation, and were associated with a hemoglobin drop of less than $2 \mathrm{~g} / \mathrm{dL}$. Since the development of the APEX trial, ISTH created a standardized definition for CRNM bleeding, which is more stringent than the APEX criteria, requiring a face-to-face evaluation. ${ }^{20}$ In contrast to CRNM bleeding, major bleeding in the APEX trial was defined according to the ISTH definition, which requires the bleed to be fatal, in a critical organ site, or be related with a hemoglobin drop of $\geq 2$ $\mathrm{g} / \mathrm{dL}^{10}{ }^{10}$ Interestingly, the ISTH criteria for major bleeding is also more sensitive than other common standardized major bleeding definitions such as thrombolysis in myocardial infarction (TIMI) major bleeding, which requires bleeds to be fatal, in a critical organ site, or associated with a hemoglobin drop of $\geq 5 \mathrm{~g} / \mathrm{dL}^{21}$

Weighing the benefit-risk of a potential bleeding event versus a potential thrombotic event is important when choosing an appropriate anticoagulant treatment and regimen. Given the increased risk of bleeding associated with extended-duration anticoagulation for the prevention of VTE in other studies, potential bleeding concerns remain a critical issue. Betrixaban did not increase major bleeds relative to enoxaparin, but it is still important to consider the increased risk in CRNM bleeding and its meaning to patients. It has previously been demonstrated that both physicians and patients considered death or a disabling stroke as the most important events to avoid, and placed little importance on the prevention of nonfatal major bleeding not requiring a transfusion, moderate bleeding, or CRNM bleeding. ${ }^{22}$ Therefore, despite an increase in CRNM bleed, physicians and patients may determine the prophylactic benefits of extended duration betrixaban may outweigh the risk of CRNM bleeding, especially considering the mild to moderate nature of the bleeds observed in the trial.

The current post hoc analyses were not prespecified and the total number of bleeding events was low. Therefore, the results should be interpreted with caution. Additionally, the severity and clinical outcomes were not adjudicated by a clinical events committee. Instead, all outcomes were determined by the site study investigators using their best clinical judgement. However, this form of evaluation best reflects the experience and assessment of these outcomes by physicians and patients in the real world.

\section{Conclusions}

Extended-duration betrixaban was associated with a twofold increase in CRNM bleeding compared with standard duration enoxaparin. The clinical consequences of bleeding were similar between treatment arms without an increase in prolonged or new hospitalizations or medical interventions. Finally, severity of bleeds was consistent between the enoxaparin and betrixaban arms, with most CRNM bleeds considered mild or moderate.

\section{Funding}

This study was funded by Portola Pharmaceuticals, Inc.

\section{Conflict of Interest}

Megan K. Yee, C. Michael Gibson, and Tarek Nafee have received consulting fees and grant support from Portola Pharmaceuticals during the conduct of the study. Gerald Chi, Adrian Hernandez, Russell Hull, and Robert Harrington have received research grant support from Portola Pharmaceuticals. Alexander Cohen has received grant support, personal fees, and nonfinancial support from Portola Pharmaceuticals during the conduct of the study. Samuel Goldhaber provided consulting for Portola Pharmaceuticals. All other authors declare no conflict of interest.

\section{References}

1 Amin AN, Varker H, Princic N, Lin J, Thompson S, Johnston S. Duration of venous thromboembolism risk across a continuum in medically ill hospitalized patients. J Hosp Med 2012;7(03): 231-238 
2 Cohen AT, Alikhan R, Arcelus JI, et al. Assessment of venous thromboembolism risk and the benefits of thromboprophylaxis in medical patients. Thromb Haemost 2005;94(04):750-759

3 Kahn SR, Lim W, Dunn AS, et al. Prevention of VTE in nonsurgical patients: Antithrombotic Therapy and Prevention of Thrombosis, 9th ed: American College of Chest Physicians Evidence-Based Clinical Practice Guidelines. Chest 2012;141(2 Suppl):e195S-e226S

4 Hull RD, Schellong SM, Tapson VF, et al; EXCLAIM (Extended Prophylaxis for Venous ThromboEmbolism in Acutely Ill Medical Patients With Prolonged Immobilization) study. Extended-duration venous thromboembolism prophylaxis in acutely ill medical patients with recently reduced mobility: a randomized trial. Ann Intern Med 2010;153(01):8-18

5 Goldhaber SZ, Leizorovicz A, Kakkar AK, et al; ADOPT Trial Investigators. Apixaban versus enoxaparin for thromboprophylaxis in medically ill patients. N Engl J Med 2011;365(23):2167-2177

6 Cohen AT, Spiro TE, Büller HR, et al; MAGELLAN Investigators. Rivaroxaban for thromboprophylaxis in acutely ill medical patients. N Engl J Med 2013;368(06):513-523

7 Spyropoulos AC, Ageno W, Albers GW, et al; MARINER Investigators. Rivaroxaban for thromboprophylaxis after hospitalization for medical illness. N Engl J Med 2018;379(12):1118-1127

8 Cohen AT, Harrington RA, Goldhaber SZ, et al; APEX Investigators. Extended thromboprophylaxis with betrixaban in acutely ill medical patients. N Engl J Med 2016;375(06):534-544

9 Cohen AT, Harrington R, Goldhaber SZ, et al. The design and rationale for the Acute Medically Ill Venous Thromboembolism Prevention with Extended Duration Betrixaban (APEX) study. Am Heart J 2014;167(03):335-341

10 Schulman S, Kearon C; Subcommittee on Control of Anticoagulation of the Scientific and Standardization Committee of the International Society on Thrombosis and Haemostasis. Definition of major bleeding in clinical investigations of antihemostatic medicinal products in non-surgical patients. J Thromb Haemost 2005;3(04):692-694

11 Cheung KS, Leung WK. Gastrointestinal bleeding in patients on novel oral anticoagulants: Risk, prevention and management. World J Gastroenterol 2017;23(11):1954-1963
12 Desai J, KolbJM, Weitz JI, AisenbergJ. Gastrointestinal bleeding with the new oral anticoagulants-defining the issues and the management strategies. Thromb Haemost 2013;110(02):205-212

13 Holster IL, Valkhoff VE, Kuipers EJ, Tjwa E. New oral anticoagulants increase risk for gastrointestinal bleeding: a systematic review and meta-analysis. Gastroenterology 2013;145(01):105-112

14 Caldeira D, Rodrigues FB, Barra M, et al. Non-vitamin K antagonist oral anticoagulants and major bleeding-related fatality in patients with atrial fibrillation and venous thromboembolism: a systematic review and meta-analysis. Heart 2015;101(15):1204-1211

15 Flaherty ML, Kissela B, Woo D, et al. The increasing incidence of anticoagulant-associated intracerebral hemorrhage. Neurology 2007;68(02):116-121

16 Dekker SE, Hoffer SA, Selman W, Bambakidis NC. Spontaneous Intracerebral Hemorrhage. Principles of Neurological Surgery. Philadelphia, PA: Elsevier Health Sciences; 2018

17 Krishnamurthi RV, Moran AE, Forouzanfar MH, et al; Global Burden of Diseases, Injuries, and Risk Factors 2010 Study Stroke Expert Group. The global burden of hemorrhagic stroke: a summary of findings from the GBD 2010 study. Glob Heart 2014;9(01):101-106

18 Fernando SM, Reardon PM, Dowlatshahi D, et al. Outcomes and costs of patients admitted to the ICU due to spontaneous intracranial hemorrhage. Crit Care Med 2018;46(05):e395-e403

19 Taylor TN, Davis PH, Torner JC, Holmes J, Meyer JW, Jacobson MF. Lifetime cost of stroke in the United States. Stroke 1996;27(09): 1459-1466

20 Kaatz S, Ahmad D, Spyropoulos AC, Schulman S; Subcommittee on Control of Anticoagulation. Definition of clinically relevant non-major bleeding in studies of anticoagulants in atrial fibrillation and venous thromboembolic disease in non-surgical patients: communication from the SSC of the ISTH.J Thromb Haemost 2015;13(11):2119-2126

21 Mehran R, Rao SV, Bhatt DL, et al. Standardized bleeding definitions for cardiovascular clinical trials: a consensus report from the Bleeding Academic Research Consortium. Circulation 2011;123 (23):2736-2747

22 Yuan Z, Levitan B, Burton P, Poulos C, Brett Hauber A, Berlin JA. Relative importance of benefits and risks associated with antithrombotic therapies for acute coronary syndrome: patient and physician perspectives. Curr Med Res Opin 2014;30(09):1733-1741 